\title{
Antimicrobial Resistance and Other Related Virulence Factors in Staphylococcus Spp isolated from Food, Environmental and Humans in Italy
}

\author{
Immacolata Anacarso ", Carla Condò, Carla Sabia, Patrizia Messi, Simona de Niederhausern, \\ Moreno Bondi, Ramona Iseppi
}

University of Modena and Reggio Emilia- Dep. of Life Sciences - Via Campi, 287 - Modena , Italy

*Corresponding Author: immacolata.anacarso@unimore.it

Copyright (C) 2013 Horizon Research Publishing All rights reserved.

\begin{abstract}
We investigated the presence of antibiotic-resistances and others important virulence factors in coagulase negative and positive staphylococci, isolated from several sources (food, environmental and clinical). Thirty-four staphylococci were isolated and identified (28 coagulase-positive were identified as Staphylococcus aureus, 6 remaining Coagulase Negative Staphylococci were identified as 3 S. epidermidis, 2 S. warneri, 1 S. hycus). All the strains were analyzed both with phenotypic and genotypic assays in order to evaluate the virulence factors and the susceptibility against antibiotics and to correlate them to the sources of isolation. Results showed that isn't a direct correlation between features researched and isolation, and the different virulence factors are as widespread as antibiotic-resistance. The isolation from food of a strain of Staphylococcus aureus with a high methicillin resistance was very interesting. 15 of the 34 strains were found to possess multi-drug resistance and in 7 cases with Minimum Inhibitory Concentration(MIC) really very high; also in this case there is no distinction between the coagulase-positive and coagulase-negative staphylococci and their origin of isolation.
\end{abstract}

Keywords Antibiotic-resistance, Virulence factors, CNS, MRSA, Staphylococci

\section{Introduction}

Staphylococci are gram-positive, non-motile, not producing spores ubiquitous bacteria that include different opportunistic/pathogenic species, responsible for human and animal infections. These groups of microorganisms colonize skin, hair, nose and throat of people and animals and from these sources they can be transferred to food because both organisms are the main reservoirs [1]. Staphylococci cause local and systemic infections in mammals ranging from minor wound infections to life-threatening conditions such as endocarditis[2], osteomyelitis [3], and toxic shock syndrome [4].

On the basis of the ability to clot blood plasma, they are divided into two groups: coagulase negative (CNS), and coagulase positive staphylococci that include Staphylococcus aureus specie.

S.aureusis a common pathogen associated with multiple disease processes, an important nosocomial and community-acquire dpathogen [5] and one of the major bacterial agents causing foodborne diseases in humans worldwide [6,7]. These microorganisms have a formidable range of potential virulence determinants [8].

Coagulasenegative staphylococci (CNS), in particular Staphylococcus epidermidis, were considered as harmless skin commensal, but in recent decades, they became important human pathogens. CNS are increasingly recognized as etiological agents of hospital acquired infections and S. epidermidis, in particular, is considered a low pathogenic microorganism, responsible for infections only in people with lowered immune system[9].

The pathogen city of both $S$. aureus and CNS, comes from their production of an impressive repertoire of virulence factors [8]that includes: surface proteins, that promote colonization of hosts tissues; invasions, that promote bacterial spread in tissue [10]; surface factors, that inhibit phagocyte engulfment [11]; biochemical proprieties that enhance their survival in phagocytes, as the catalase production; immunological disguises and membrane-damaging toxins that lyse eukaryotic cell membranes [12]; exotoxins that damage host tissues or otherwise provoke symptoms of disease [8]. An emerging problem is also represented by the community-associated multi-resistant $S$. aureus infections[13]. During the past 15 years, the prevalence of methicillin-resistant Staphylococcus aureus(MRSA) is increased in many parts of the world [14]causing MRSA infections such as bacteremia often associated to a high mortality [15]. The causes of the multidrug resistant strains emergence are mainly to be found 
in excessive and/or inappropriate use of antibiotics and their use in animal husbandry. The use of antibiotics in livestock not only for therapeutic purposes, but also for preventive and auxinicscope is, in fact, largely responsible for the selection and spread of antimicrobial-resistant microorganisms in the environment, which can reach humans through the food chain. To make matters worse, sometimes genes for resistance and virulence can be transferred together, leading to the emergence of new resistance threats of greater virulence and pathogenicity than seen in past generations (superbugs).

With regard toS. epidermidisnot much is known about mechanisms of pathogenesis[16]. A characteristic found in many pathogenic strains is the production of slime, resulting in biofilm formation. The slime is predominantly a secreted teichoic acid, normally found in the cell wall of the staphylococci. Slime production, with the ability to form biofilm on various surfaces, is considered a significant virulence factor for some staphylococci isolated from clinical samples [17, 18].This characteristic and the capability to acquire antibiotic-resistant genes are probably a significant determinant of virulence in this specie also [19].

The aim of this work is to study staphylococci strains, both coagulase-positive and coagulase-negative, isolated from several sources (food, environmental and clinical samples) evaluating the main virulence factors and antibiotic-resistances. The possible correlation between all these virulence factors and sources of bacterial isolation was also investigated.

\section{Materials and Methods}

\subsection{Bacterial Strain Identifications}

A total of 34 strains from food environmental and clinical sources were tested, both with phenotypic and genotypic assays to research the main virulence factors and the antibiotic-resistance.

Strains found in food have been isolated from egg pasta, custard cream, meat and pastries bought in a supermarket of the large-scale distribution.

Strains of environmental and clinical sources have been isolated from air valuations of university rooms and from swabs of hospitalized patients.

All the strains were isolated on Mannitol Salt Agar(bioMerieux, Italy). They were further analyzed for the presence of coagulase activity (Pastore ${ }^{\mathrm{TM}}$ Staph-Plus BIO RAD). The strains are finally speciated by VITEK 2 Gram Positive (GP) identification card (Product number 21342) (bioMérieux, Italy).

\subsection{Phenotypic Characterization}

All tests were repeated three times.

\subsubsection{Determination of Hemolysins Production}

All 34 Staphylococci were first cultivated in Brain Heart Infusion agar (BHI - Difco Laboratories, Detroit, MI) and incubated at $37{ }^{\circ} \mathrm{C}$ overnight. The strains were passed on blood agar plates and incubate at $37^{\circ} \mathrm{C}$ for $24 \mathrm{~h}$. The Blood agar had a volume of $15 \mathrm{ml}$ and was prepared with Difco ${ }^{\mathrm{TM}}$ Tryptose Blood Agar Base (Becton Dickinson) and supplemented with $7 \%$ sterile defibrinated sheep blood (Biomerieux, Italy). A positive result was indicated by the formation of a clear zone of haemolysis ( $\beta$-haemolysis), a partial and greening haemolysis zone ( $\alpha$-haemolysis), or no activity ( $\gamma$-haemolysis) around the spots.

\subsubsection{Determination of Lipase and Lecithinase}

Lipolytic activity was determined on plates containing $2 \%$ agar base, $1 \%$ peptone, $1 \%$ yeast extract, $0,1 \% \mathrm{CaCl}_{2}$ and $2 \%$ Tween 80 . A positive result was defined by the formation of opacity around the colonies after incubation at $37^{\circ} \mathrm{C}$ for $24 \mathrm{~h}$ shown in Figure $2 b$. The production of lecithinase was studied in Baird-Parker agar (Oxoid, Italy). A positive result was indicated by the formation of an opaque halo around the colonies after incubation at $37^{\circ} \mathrm{C}$ for $48 \mathrm{~h}$.

\subsubsection{Determination of Dnase and Thermonuclease Tests}

DNAse was determined on DNAse agar (BD Diagnostic Systems). After incubation at $37^{\circ} \mathrm{C}$ for $24 \mathrm{~h}$, plates were covered with $\mathrm{HCl}$, a positive result was defined by the formation of opacity around the colonies. Thermonuclease test (T-test) was determined in Thermonuclease Agar w/toluidine blue (Thermo Scientific, Milan, Italy). The production of thermonuclease was indicated by the formation of pink colonies after incubation at $37^{\circ} \mathrm{C}$ for $24 \mathrm{~h}$ following by incubation at $100^{\circ} \mathrm{C}$ for $10 \mathrm{~min}$. For both tests Staphylococcus aureus ATCC 25923 and Staphylococcus epidermidis ATCC 12228 as positive and negative control, respectively, were used.

\subsubsection{Determination of Hyaluronidase}

The presence ofhyluronidase was evaluated on Brain Heart Infusion agar (BHI - Difco Laboratories, Detroit, MI) containing hyaluronic acid $(0,4 \mathrm{mg} / \mathrm{ml})$ as described elsewhere [1]. After incubation at $37^{\circ} \mathrm{C}$ for $24 \mathrm{~h}$, plates were covered with cetylpyridinium chloride and a positive result was indicated by the formation of a transparent halo around the colonies in Figure 2c.

\subsubsection{Determination of Gelatinase}

Gelatinaseproduction was assessed by inoculation of the strains in a Nutrient broth containing $10 \%$ gelatin, which was then incubated at $37^{\circ} \mathrm{C}$ for $48-72 \mathrm{~h}$. Positive gelatinase was recorded as degradation of the gelatin to liquid.

\subsubsection{Study of Slime Production}

Slime production was evaluated by Congo red agar method. The medium was prepared with $37 \mathrm{~g} / \mathrm{l}$ Brain Heart Infusion broth (BHI - Difco Laboratories, Detroit, MI), $50 \mathrm{~g} / 1$ sucrose, $10 \mathrm{~g} / \mathrm{l}$ agar and $0,8 \mathrm{~g} / \mathrm{l}$ Congo red [17]. Plates were incubated at $37^{\circ} \mathrm{C}$ for $24 \mathrm{~h}$ and then incubated at room 
temperature for $12 \mathrm{~h}$. A positive result was indicated by black colonies on the surface, shown in Figure 2a.

\section{Genotypic Characterization}

DNA was extracted from about 10 single colonies with the DNeasy tissue kit (Qiagen, Milan, Italy) as specified by the manufacturer and using lysostaphin $(100 \mu \mathrm{g} / \mathrm{ml}$; Sigma, Italy) to achieve bacterial lysis. The concentration of DNA was assessed spectrophotometrically. The genetic determinants for the following virulence factors were investigated using multiplex PCR: staphylococcal enterotoxins $(s e a-e)$,toxic shock syndrome toxin (tst) and alpha/beta-hemolysins ( $h l a$ and $h l b$ ) [8], coagulase (coa) [20],aureolysin (aur) [21], fibronectin binding proteins $\mathrm{A}$ and $\mathrm{B}(f n b A$ and $f n b B)[8]$, adhesins Map/Eap (Map/Eap) [12]. PCR amplifications were performed with Ready-to-Go-PCR beads (Amersham Pharmacia Biotech, Freiburg, Germany) in a $25-\mu 1$ reaction mixture containing approximately $10 \mathrm{ng}$ of template DNA and $2.5 \mathrm{pmol}$ of each primer. The PCR condition are shown in Table 3. The PCR products were analyzed on a $1 \%$ agarose gel and further controlled by sequencing.

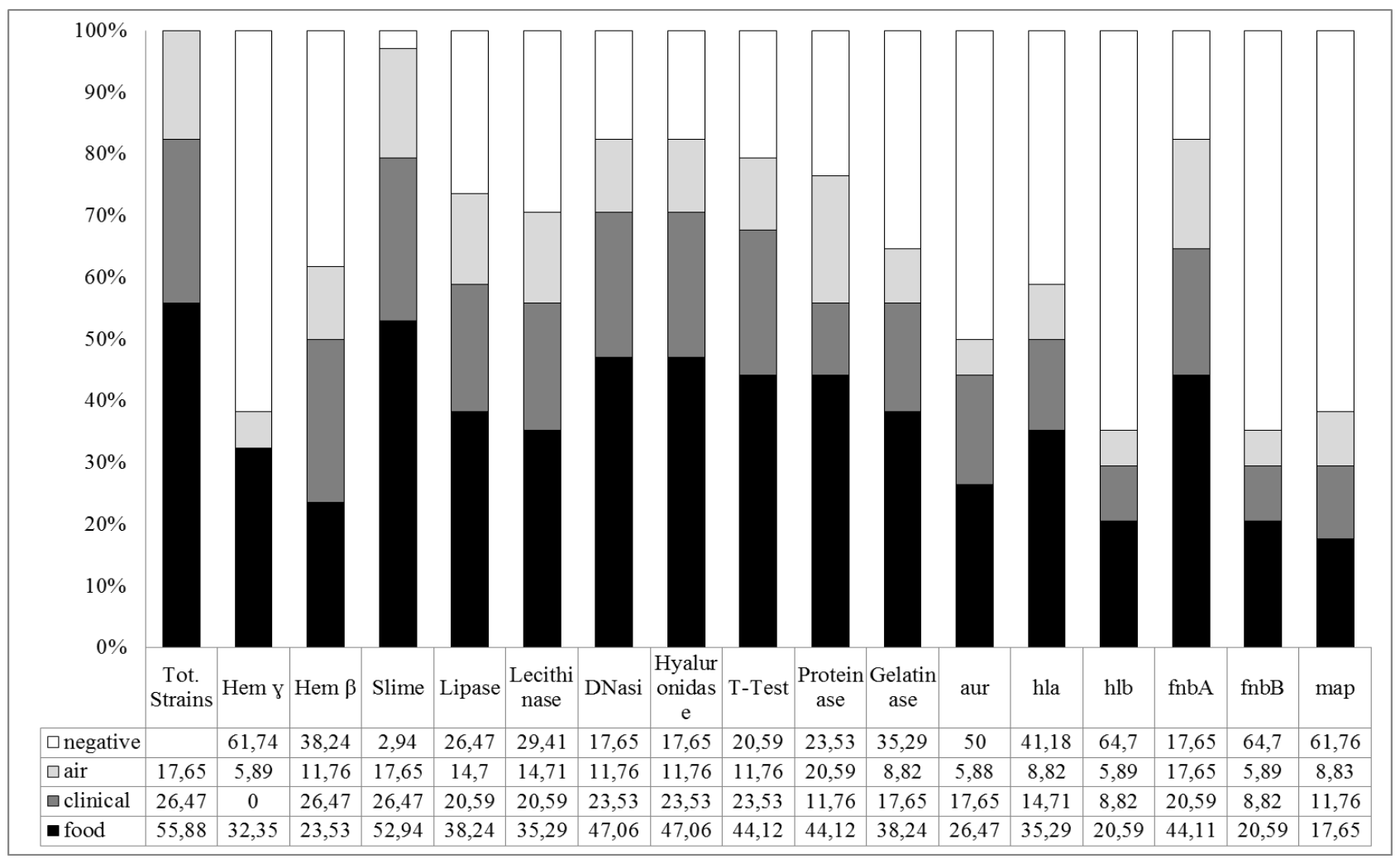

Figure 1. Phenotipic and genotypic results in percentage form
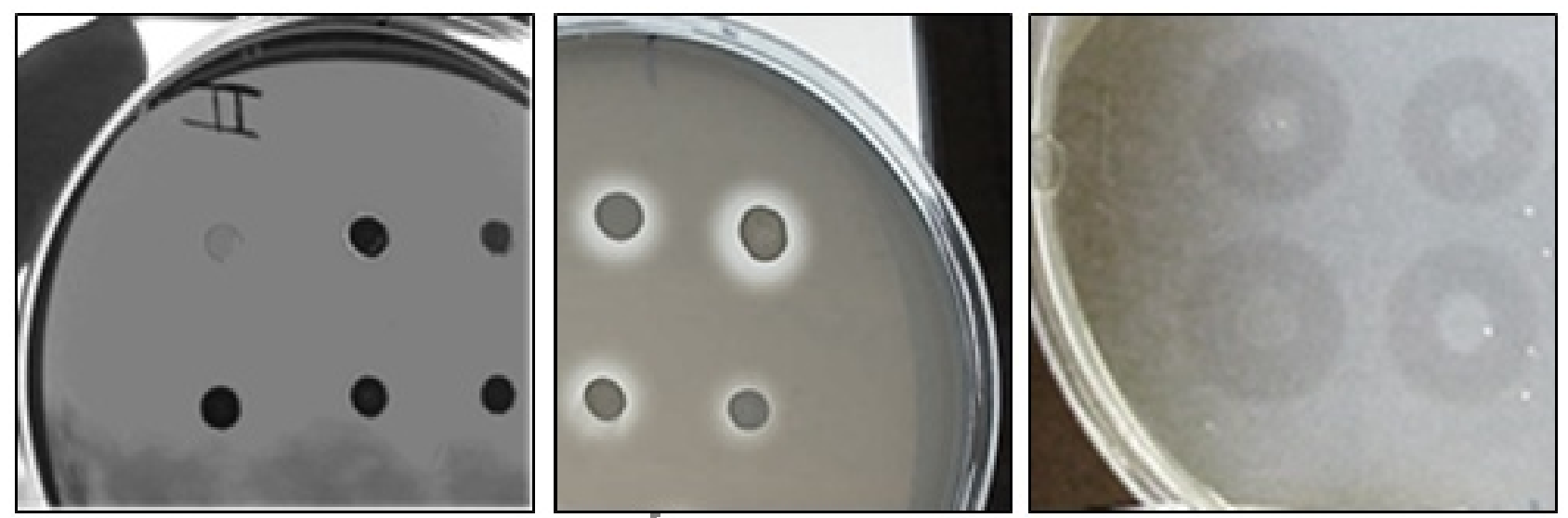

Examples of the resultsphenotypic: left slime test, center and right respectively DNase and production of Hyaluronidase

Figure 2. Phenotipic results 
Table 1. Phenotipic and genotypic results

\begin{tabular}{|c|c|c|c|c|c|c|c|c|c|c|c|c|c|c|c|}
\hline STRAINS & $\mathrm{He}$ & $\mathbf{S}$ & $\mathbf{L i}$ & Le & D & Hy & TT & $\mathbf{G}$ & $\mathbf{P}$ & au & hA & hB & fA & fB & ma \\
\hline *STAM 2TIEN - a & $\beta$ & + & + & + & + & + & + & - & - & + & + & - & + & - & - \\
\hline *STAM 4 TIEN - f & $\beta$ & + & + & + & + & + & + & + & + & + & + & + & + & + & + \\
\hline **STM 7 TIEN - f & $\gamma$ & + & + & - & - & - & - & - & + & - & - & - & - & - & - \\
\hline *STAM 15 TIEN - f & $\boldsymbol{\beta}$ & + & + & + & + & + & + & + & + & - & + & - & + & - & + \\
\hline$*$ STAM $1 \mathrm{~A}-\mathrm{c}$ & $\boldsymbol{\beta}$ & + & + & + & + & + & + & + & + & + & - & - & + & - & - \\
\hline$*$ STAM $2 \mathrm{~A}-\mathrm{f}$ & $\beta$ & + & + & + & + & + & + & + & + & - & + & - & - & - & + \\
\hline$*$ STAM 3A - f & $\beta$ & + & + & + & + & + & + & + & + & + & - & - & + & - & - \\
\hline$*$ STAM $4 \mathrm{~A}-\mathrm{f}$ & $\beta++$ & + & + & + & + & + & + & + & + & - & - & + & + & + & + \\
\hline$*$ STAM $11 \mathrm{~A}-\mathrm{a}$ & $\boldsymbol{\beta}$ & + & + & + & + & + & + & + & + & + & - & - & + & - & + \\
\hline$* *$ STM $12 \mathrm{~A}-\mathrm{f}$ & $\gamma$ & + & - & - & - & - & - & - & + & - & - & - & + & - & - \\
\hline$*$ STAM $15 \mathrm{~A}-\mathrm{f}$ & $\gamma$ & + & - & - & + & + & + & + & + & + & - & + & + & + & - \\
\hline$* *$ STM $16 \mathrm{~A}-\mathrm{a}$ & $\beta$ & + & - & - & - & - & - & - & - & - & - & + & + & + & - \\
\hline *STAM 17A - f & $\beta++$ & - & + & + & + & + & + & + & + & - & + & - & + & - & - \\
\hline *STAM $20-\mathrm{c}$ & $\beta$ & + & - & + & + & + & + & + & - & + & + & + & + & + & + \\
\hline *STAM $22-\mathrm{f}$ & $\beta$ & + & + & + & + & + & + & + & + & + & + & + & + & + & - \\
\hline *STAM $416-\mathrm{f}$ & $\gamma$ & + & + & + & + & + & + & + & + & + & + & - & + & - & + \\
\hline$*$ STAM $616-\mathrm{f}$ & $\gamma$ & + & + & + & + & + & + & + & + & + & + & - & + & - & + \\
\hline *STAM $620-\mathrm{c}$ & $\beta$ & + & + & - & + & + & + & + & - & + & + & - & + & - & + \\
\hline *STAM-BP 2 - a & $\gamma$ & + & + & + & + & + & + & + & + & - & + & - & + & - & + \\
\hline *STAM-BP $4-\mathrm{f}$ & $\gamma$ & + & + & - & + & + & - & + & - & + & + & + & + & + & - \\
\hline *STAM-BP $5-\mathrm{f}$ & $\gamma$ & + & + & + & + & + & + & - & + & - & - & - & + & - & - \\
\hline *STAM-BP 7 - f & $\beta$ & + & + & + & + & + & + & + & + & - & + & - & + & - & - \\
\hline *STAM-BP $789-\mathrm{f}$ & $\gamma$ & + & - & + & + & + & + & + & + & + & + & - & + & - & - \\
\hline *STAM-BP 3219 - f & $\gamma$ & + & - & - & + & + & + & - & - & + & + & + & + & + & - \\
\hline *STAM-BP $117-\mathrm{f}$ & $\gamma$ & + & - & - & + & + & + & - & - & - & + & + & - & + & - \\
\hline *STAM 359 A - c & $\beta$ & + & + & + & + & + & + & - & + & - & + & - & + & - & + \\
\hline *STAM 386 A - c & $\beta$ & + & + & + & + & + & + & + & + & + & - & - & - & - & - \\
\hline *STAM 594 A - c & $\beta$ & + & + & + & + & + & + & - & + & - & + & + & + & + & + \\
\hline *STAM $653 \mathrm{~A}-\mathrm{c}$ & $\beta$ & + & + & + & + & + & + & + & + & + & - & - & + & - & - \\
\hline **STM $95 \mathrm{C}-\mathrm{a}$ & $\gamma$ & + & + & + & - & - & - & - & + & - & - & - & + & - & - \\
\hline *STAM 98 D - a & $\beta$ & + & + & + & + & + & + & + & + & - & + & + & + & + & + \\
\hline *STAM T $17-\mathrm{c}$ & $\beta$ & + & + & + & + & + & + & + & + & + & + & + & - & + & - \\
\hline **STM $28-\mathrm{c}$ & $\beta++$ & + & - & - & - & - & - & - & + & - & - & - & + & - & - \\
\hline
\end{tabular}

Phenotipic tests (He: Hemolysins; S: Slime; Li: Lipase; Le: Lecithinase; D: DNase; Hy: Hyaluronidase; TT: Thermonuclease;

G: Gelatinase; P: Proteinase).

Genotipic test s (Au: aureolisin; hA: hla; hB: hlb; fA: fnbAfB: fnbB; ma: map/eap).

f: foodisolation; c: clinicalisolation; a: air isolation

*Staphylococcicoagulase-positive; ** Staphylococcicoagulase-negative +: positive result; - : negative results 
Table 2. Primers and amplification conditions

\begin{tabular}{|c|c|c|c|c|}
\hline Target gene & Forwardsequence & Reverse sequence & Lenght & PCR conditions \\
\hline sea & $\begin{array}{l}\text { AAA GTC CCC ATC } \\
\text { AAT TTA TGG CTA }\end{array}$ & $\begin{array}{l}\text { GAAC CGA AGG TTC } \\
\text { TGT AGA }\end{array}$ & $216 \mathrm{bp}$ & $\begin{array}{c}94^{\circ} \mathrm{C}\left(1^{\prime}\right)-\left\{\left[92^{\circ} \mathrm{C}\left(45^{\prime \prime}\right)-50^{\circ} \mathrm{C}\left(45^{\prime \prime}\right)-72^{\circ} \mathrm{C}\left(45^{\prime \prime}\right)\right] 30\right. \\
\text { cycles }\}-72^{\circ} \mathrm{C}\left(10^{\prime}\right)\end{array}$ \\
\hline$s e b$ & $\begin{array}{l}\text { TCG CAT CAA ACT } \\
\text { GAC AAA CG }\end{array}$ & $\begin{array}{l}\text { GCA GGT ACT CTA } \\
\text { TAA GTG CC }\end{array}$ & 478 bp & $\begin{array}{c}94^{\circ} \mathrm{C}\left(1^{\prime}\right)-\left\{\left[92^{\circ} \mathrm{C}\left(45^{\prime \prime}\right)-55^{\circ} \mathrm{C}\left(45^{\prime \prime}\right)-72^{\circ} \mathrm{C}\left(45^{\prime \prime}\right)\right] 30\right. \\
\text { cycles }\}-72^{\circ} \mathrm{C}\left(10^{\prime}\right)\end{array}$ \\
\hline $\mathrm{sec}$ & $\begin{array}{l}\text { GAC ATA AAA GCT } \\
\text { AGG AAT TT }\end{array}$ & $\begin{array}{l}\text { AAA TCG GAT TAA } \\
\text { CAT TAT CC }\end{array}$ & $257 \mathrm{bp}$ & $\begin{array}{c}94^{\circ} \mathrm{C}\left(1^{\prime}\right)-\left\{\left[92^{\circ} \mathrm{C}\left(45^{\prime \prime}\right)-46^{\circ} \mathrm{C}\left(45^{\prime \prime}\right)-72^{\circ} \mathrm{C}\left(45^{\prime \prime}\right)\right] 30\right. \\
\text { cycles }\}-72^{\circ} \mathrm{C}\left(10^{\prime}\right)\end{array}$ \\
\hline sed & $\begin{array}{l}\text { CTA GTT TGG TAA } \\
\text { TAT CTC CT }\end{array}$ & $\begin{array}{l}\text { TAA TGC TAT ATC } \\
\text { TTA TAG GG }\end{array}$ & $317 \mathrm{bp}$ & $\begin{array}{c}94^{\circ} \mathrm{C}\left(1^{\prime}\right)-\left\{\left[92^{\circ} \mathrm{C}\left(45^{\prime \prime}\right)-50^{\circ} \mathrm{C}\left(45^{\prime \prime}\right)-72^{\circ} \mathrm{C}\left(45^{\prime \prime}\right)\right] 30\right. \\
\text { cycles }\}-72^{\circ} \mathrm{C}\left(10^{\prime}\right)\end{array}$ \\
\hline see & $\begin{array}{l}\text { TAG ATA AGG TTA } \\
\text { AAA CAA GC }\end{array}$ & $\begin{array}{l}\text { TAA CTT ACC GTG } \\
\text { GAC CCT TC }\end{array}$ & $170 \mathrm{bp}$ & $\begin{array}{c}94^{\circ} \mathrm{C}\left(1^{\prime}\right)-\left\{\left[92^{\circ} \mathrm{C}\left(45^{\prime \prime}\right)-50^{\circ} \mathrm{C}\left(45^{\prime \prime}\right)-72^{\circ} \mathrm{C}\left(45^{\prime \prime}\right)\right] 30\right. \\
\text { cycles }\}-72^{\circ} \mathrm{C}\left(10^{\prime}\right)\end{array}$ \\
\hline tst & $\begin{array}{l}\text { ATG GCA GCA TCA } \\
\text { GCT TGA TA }\end{array}$ & $\begin{array}{l}\text { TTT CCA ATA ACC } \\
\text { ACC CGT TT }\end{array}$ & $350 \mathrm{bp}$ & $\begin{array}{c}94^{\circ} \mathrm{C}\left(1^{\prime}\right)-\left\{\left[92^{\circ} \mathrm{C}\left(1^{\prime}\right)-55^{\circ} \mathrm{C}\left(1^{\prime}\right)-72^{\circ} \mathrm{C}\left(1^{\prime}\right)\right] 30 \text { cycles }\right\}- \\
72^{\circ} \mathrm{C}\left(10^{\prime}\right)\end{array}$ \\
\hline aur & $\begin{array}{l}\text { CCT CCT GAA ATC } \\
\text { TTA AAA ACA GC }\end{array}$ & $\begin{array}{l}\text { CGA TTA TTG CGT } \\
\text { CTT ACA TAG }\end{array}$ & $346 \mathrm{bp}$ & $\begin{array}{c}94^{\circ} \mathrm{C}\left(1^{\prime}\right)-\left\{\left[92^{\circ} \mathrm{C}\left(45^{\prime \prime}\right)-50^{\circ} \mathrm{C}\left(45^{\prime \prime}\right)-72^{\circ} \mathrm{C}\left(45^{\prime \prime}\right)\right] 30\right. \\
\text { cycles }\}-72^{\circ} \mathrm{C}\left(10^{\prime}\right)\end{array}$ \\
\hline coa & $\begin{array}{l}\text { ATA GAG ATG CTG } \\
\text { GTA CAG G }\end{array}$ & $\begin{array}{l}\text { GCT TCC GAT TGT } \\
\text { TCG ATG C }\end{array}$ & Polimor-fism & $\begin{array}{c}94^{\circ} \mathrm{C}\left(1^{\prime}\right)-\left\{\left[92^{\circ} \mathrm{C}\left(1^{\prime}\right)-55^{\circ} \mathrm{C}\left(1^{\prime}\right)-72^{\circ} \mathrm{C}\left(1^{\prime}\right)\right] 30 \text { cycles }\right\}-72^{\circ} \\
\mathrm{C}\left(10^{\prime}\right)\end{array}$ \\
\hline hla & $\begin{array}{l}\text { GGT TTA GCC TGG } \\
\text { CCT TC }\end{array}$ & $\begin{array}{l}\text { CAT CAC GAA CTC } \\
\text { GTT CG }\end{array}$ & 534 bp & $\begin{array}{c}94^{\circ} \mathrm{C}\left(1^{\prime}\right)-\left\{\left[92^{\circ} \mathrm{C}\left(45^{\prime \prime}\right)-48^{\circ} \mathrm{C}\left(45^{\prime \prime}\right)-72^{\circ} \mathrm{C}\left(45^{\prime \prime}\right)\right] 30\right. \\
\text { cycles }\}-72^{\circ} \mathrm{C}\left(10^{\prime}\right)\end{array}$ \\
\hline$h l b$ & $\begin{array}{l}\text { GCC AAA GCC GAA } \\
\text { TCT AAG }\end{array}$ & $\begin{array}{l}\text { GCG ATA TAC ATC } \\
\text { CCA TGG C }\end{array}$ & 833 bp & $\begin{array}{c}94^{\circ} \mathrm{C}\left(1^{\prime}\right)-\left\{\left[92^{\circ} \mathrm{C}\left(45^{\prime \prime}\right)-55^{\circ} \mathrm{C}\left(45^{\prime \prime}\right)-72^{\circ} \mathrm{C}\left(45^{\prime \prime}\right)\right] 30\right. \\
\text { cycles }\}-72^{\circ} \mathrm{C}\left(10^{\prime}\right)\end{array}$ \\
\hline$f n b A$ & $\begin{array}{l}\text { GCG GAG ATC AAA } \\
\text { GAC AA }\end{array}$ & $\begin{array}{l}\text { CCA TCT ATA GCT } \\
\text { GTG TGG }\end{array}$ & 1278 bp & 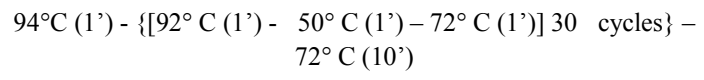 \\
\hline$f n b B$ & $\begin{array}{l}\text { GGA GAA GGA ATT } \\
\text { AAG GCG }\end{array}$ & $\begin{array}{l}\text { GCC GTC GCC TTG } \\
\text { AGC GT }\end{array}$ & 812 bp & $\begin{array}{c}94^{\circ} \mathrm{C}\left(1^{\prime}\right)-\left\{\left[92^{\circ} \mathrm{C}\left(1^{\prime}\right)-50^{\circ} \mathrm{C}\left(1^{\prime}\right)-72^{\circ} \mathrm{C}\left(1^{\prime}\right)\right] 30 \text { cycles }\right\}-72^{\circ} \\
\mathrm{C}\left(10^{\prime}\right)\end{array}$ \\
\hline map-eap & $\begin{array}{l}\text { TAA CAT TTA ATA } \\
\text { AGA ATC AA }\end{array}$ & $\begin{array}{l}\text { CCA TTT ACT GCA } \\
\text { ATT GT }\end{array}$ & $943-949$ bp & $\begin{array}{c}94^{\circ} \mathrm{C}\left(1^{\prime}\right)-\left\{\left[92^{\circ} \mathrm{C}\left(1^{\prime}\right)-45^{\circ} \mathrm{C}\left(1^{\prime}\right)-72^{\circ} \mathrm{C}\left(1^{\prime}\right)\right] 30 \text { cycles }\right\}-72^{\circ} \\
\mathrm{C}\left(10^{\prime}\right)\end{array}$ \\
\hline mecA & $\begin{array}{l}\text { AAA ATC GAT GGT } \\
\text { AAA GGT TGG C }\end{array}$ & $\begin{array}{l}\text { AGT TCT GCA GTA } \\
\text { CCG GAT TTG C }\end{array}$ & 314 bp & $\begin{array}{c}94^{\circ} \mathrm{C}\left(3^{\prime}\right)-\left\{\left[94^{\circ} \mathrm{C}\left(30^{\prime \prime}\right)-55^{\circ} \mathrm{C}\left(30^{\prime \prime}\right)-72^{\circ} \mathrm{C}\left(30^{\prime \prime}\right)\right] 30\right. \\
\text { cycles }\}-72^{\circ} \mathrm{C}\left(10^{\prime}\right)\end{array}$ \\
\hline
\end{tabular}


Table 3. MinimalConcentrationInhibition (MIC) for $\mu \mathrm{g}-\mathrm{ml}$

\begin{tabular}{|c|c|c|c|c|c|}
\hline Strain & Oxacyllin & Amikacin & Erythromycin & Rifampin & Tetracycline \\
\hline *STAM 2TIEN- a & $\mathrm{S}$ & S & $\mathrm{S}$ & $8 \gamma$ & $\mathrm{S}$ \\
\hline *STAM 1A- c & S & S & S & $8 \gamma$ & S \\
\hline *STAM 11A-a & $\mathrm{S}$ & $\mathrm{S}$ & S & $4 \gamma$ & S \\
\hline *STAM 15A - f & $>64 \gamma$ & $>2048 \gamma$ & S & $2 \gamma$ & $128 \gamma$ \\
\hline *STAM 20 - c & S & $\mathrm{S}$ & S & $4 \gamma$ & S \\
\hline *STAM 620 - c & S & S & S & $4 \gamma$ & S \\
\hline *STAM-BP 4 - f & $\mathrm{S}$ & $>2048 \gamma$ & $\mathrm{S}$ & $2 \gamma$ & S \\
\hline *STAM-BP 7 - f & $\mathrm{S}$ & $\mathrm{S}$ & $8 \gamma$ & $\mathrm{S}$ & $\mathrm{S}$ \\
\hline *STAM-BP 789 - f & S & S & S & $4 \gamma$ & S \\
\hline *STAM 359 A - c & S & $16 \gamma$ & S & $>128 \gamma$ & S \\
\hline *STAM 386 A - c & $\mathrm{S}$ & $2048 \gamma$ & $\mathrm{S}$ & $>128 \gamma$ & $\mathrm{S}$ \\
\hline *STAM 594 A - c & $\mathrm{S}$ & $16 \gamma$ & $\mathrm{S}$ & $>128 \gamma$ & $\mathrm{S}$ \\
\hline **STM 95 C - a & $\mathrm{S}$ & $64 \gamma$ & $>128 \gamma$ & $>128 \gamma$ & $\mathrm{S}$ \\
\hline *STAM 98 D - a & S & $64 \gamma$ & S & $>128 \gamma$ & S \\
\hline **STM $28-\mathrm{c}$ & $\mathrm{S}$ & S & $>128 \gamma$ & $2 \gamma$ & S \\
\hline
\end{tabular}

f: foodisolation, c: clinicalisolation, a: air isolation

* Staphylococcicoagulase-positive; ** Staphylococcicoagulase-negative

$\gamma$ : $\mu \mathrm{g}-\mathrm{ml} \mathbf{S}$ : antibiotic sensible

\section{Antibiotic-Resistance}

Assessments were repeated three time.

All strains were tested for their antibiotic susceptibility profiles by the broth microdilution method against the following drugs: oxacillin, erythromycin, vancomycin, teicoplanin, kanamycin, amikacin, tetracycline, rifampicin (Sigma Aldrich, Milan, Italy) and daptomycin (Novartis Pharma, Basel, Switzerland) in accordance with Clinical Laboratory Standards Institute guidelines 2012 [22]. The European Committee on Antimicrobial Susceptibility Testing guidelines were used for the susceptibility categorization [23]. Quality control strains included $S$. aureus ATCC 29213.

The research of the gene mec $A$ (methicillin resistance) was also analyzed by PCR reaction [12] using the conditions listed in Table 3.

\section{Results}

\subsection{Bacterial Strains Identification}

Of the 34strains, twenty-eight were identified as Staphylococcus aureus, three S. epidermidis, two $S$. warneri and one S. hyicus. Among all strains the $55.88 \%$ were from food, $26,47 \%$ of clinical origin and $17.65 \%$ environmental.

In particular three of the CNS were isolated from food sources, two from air and one was a clinical isolation.

Among S. aureus, eightstrains were isolated from clinical sources, four from air and sixteen from food.

\subsection{Phenotypic Characterization}

Sixteenof twenty-eight $S$. aureus were positive for all phenotypic tests, and the remaining twelve were positive for two or more tests.

In particular about the clinical strains, $26,47 \%$ were at the same time with a $\beta$-hemolytic profile and positivity at the slime. $23,53 \%$ of the same strains presented simultaneously positivity to DNase, hyaluronidase and thermonuclease; the simultaneous positivity for these tests was also found in strains isolated from the air at $11,76 \%$, and almost all those isolated from foods, positive for DNase and T-Test at $47,06 \%$ and positive forhyaluronidase at percentage to $43 \%$.

All results in percentage form are reported in Figure 1.

Regarding CNS, all strains resulted positive for slime production, with the exception of the STM-BP1 strain. Three CNS strains were noteworthy: STM95C, was found positive forlipase, lecithinase and protease; STM16A, has shown a marked $\beta$-hemolytic action, as STM28, that has exhibited an high proteolytic activity also.

All twenty-eight Staphylococcus aureus strains showed positivity to hyaluronidase; 22 of these were positive for protease, concurrent positive for gelatinase in 19 cases.

\subsection{Genotypic Characterization}

Primers used and amplification conditions are shown in Table 2.All S. aureus strains were found positive for 
coa(data not shown). Seventeen $(60 \%)$, twenty (71\%), nine $(32 \%)$, twenty-four $(85 \%)$, eleven $(39 \%)$ and thirteen $(46 \%)$ were found positive, to aur, hla, hlb, fnbA, fnbB and map/eap respectively. With regard to the staphylococcal enterotoxins, in only 10 cases the amplification was obtained for one or more genes involved. In particular sea was detected in only two strains, seb and sec, were detected in six and four strains respectively. There were no amplification for sed, see and tst. All PCR reactions were found negative for CNS, with the exception off our strains, in which $f n b A$ and $f n b B$, genes coding for the fibronectin binding proteins $\mathrm{A}$ and $\mathrm{B}$, were present both or individually.

All results of phenotypic and genotypic tests are reported in Table 1 and in Figure 1.

\subsection{Antibiotic-Resistance}

Of34analyzed strains, fifteen(44\%) were resistant to one or more antibiotics. 4 strains, amount the $11,76 \%$ were isolated from air and as many from food; 7 equal to $20,59 \%$ were clinical isolated. For these resistant strains was made the Minimum Inhibitory Concentration (MIC), the results of which are indicated in Table 3.Of these strains, except one, showed resistance to rifampicin and five of these with very high levels, with MIC of $128 \mu \mathrm{g} / \mathrm{ml}$.

7 strains showed resistance to amikacin, five with high levels, with MIC of $64 \mu \mathrm{g} / \mathrm{ml}$ or $2048 \mu \mathrm{g} / \mathrm{ml}$. Also for the three strains resistant to erythromycin in two cases the levels of resistance were high, as well as for the single strain resistant to tetracycline and the only one to oxacillin.

4 strains have shown the simultaneous resistance to two or three antibiotics, among these the strain STAM15A, a food isolated $S$. aureus, the only with resistance to oxacillin and contextual resistance to amikacin and tetracycline and the strain STM95C, a coagulase-negative strain from air, both with very high levels of resistance equal to or greater than $64 \mu \mathrm{g} / \mathrm{ml}$.

\section{Discussion}

A direct relationship between bacterial virulence factors and sources of isolation did not emerged in the present investigation. In this study we noted that all $S$. aureus strains are endowed with a lot of virulence factors, feature much less represented among the CNS isolates that however were all positive for the production of slime. This is a substance that helps the microorganism in the production of biofilm, a consortium of microorganisms in which all the species can survive in adverse conditions, resulting also resistant to cleaning agents or drugs. For this reason biofilm formation has been recognized as an important virulence factor. Our data on the biofilm formation potentiality are difficult to compare with those of others Authors, because this feature has been prevalently studied in clinical isolated strains. Ishak et al. report 13 of 14 clinical isolated staphylococci with slime production [24].Kotilainenrefers that $34(53 \%)$ of 46 strains from adult septicemia were adherent slime producers [25]. Overall, others Authors have also shown that the proportion of slime producing clinical isolated CNS strains can vary from $20 \%$ to $89 \%[6,26]$. In the present study 6 of $6(100 \%)$ of coagulase-negative staphylococci isolated were slime positive, and this capability was noted in all the strains, despite the different origin, mainly from food and environment origin.

With regard to S.aureus, all strainswere positive to hyaluronidase and DNase assays. The presence or absence of hyaluronidase among staphylococci has been examined by other authors. Choudhuri and Chakrabarty, report that only $13 \%$ of 523 staphylococcus, of which $96 \%$ coagulase positive, isolates from human pathologies, were negative for hyaluronidase activity [27].

Essers and Redebold, showed of 218 coagulase positive staphylococci, isolated from human specimens, that only one isolate was hyaluronidase negative [28]. The same authors report in their study staphylococci with the ability to produce, at the same time, the DNAse and hyaluronidase activity. In particular hyaluronidase is predominantly seen among strains of staphylococci that exibit both coagulase and DNAse activity.

In the present study, 28 of $28(100 \%)$ coagulase-positive staphylococci, identified as Staphylococcus aureus, were hyaluronidase positive and, contextually, showed the ability toproduce DNAse. Our results are partly agreed with the data of the Authors cited above; no study has highlighted a rate of $100 \%$ related to the simultaneous presence of hyaluronidase and DNAse activities. This higher percentagemay be due to the smaller number of strains employed in the present study but, despite this limitation, the results allow us to point out that the virulence factors, that characterize staphylococci, are equally distributed in nature, without differences due to the origin.

At the same time, we haven't found significant differences in the distribution of antibiotic resistances among the isolates of different origins. In particular, of fifteen resistant staphylococci, including two CNS strains, seven were of clinical origin, four were isolated from air and four from food, and all were endowed with high level of resistance. This demonstrates that, irrespective of the source of isolation or species, strains may be equally hazardous. Virulence factors were also found in strains of coagulase-negative staphylococci, demonstrating once again how these microorganisms may represent hazards and behave as true pathogens in determinate conditions, in particular when the host is immune-compromised. In conclusion, although on the basis of previous studies it is right to expect that strains of clinical origin may have characteristics of virulence more enhanced than those isolated from food or environment, our results suggest that also microorganisms of non-clinical sources, can be equally virulent and a danger to human health. In this investigation, most isolates are food-borne or environmental strains and they do not differ for the presence of virulence factors and antibiotic resistance from strains of clinical origin. To support what above discussed, we also 
underline that the only resistance to oxacillin, with the mecA gene discovered, was found in a S.aureus strain isolated from food, coding for high levels of resistance, an evidence of the wide spread of this trait out of hospitals, as reported from other authors [29, 30].Staphylococcus is a genus more and more able to acquire virulence factors and new antibiotic resistance genes. In a previous work we demonstrated the horizontal passage of vancomycin resistance determinants from enterococci to two $S$. aureus strains transfer confirmed by vanA gene amplification in both transconjugants [8]. Considering the environmental diffusion of $S$. aureus and the increasingly close link with food, these microorganisms pose additional food safety and occupational health concerns. In particular, infected food handlers may contaminate food of animal origin during slaughter and processing of livestock, or by cross-contamination during food preparation. Antibiotic resistance and other virulence factors are common in staphylococci, and during the mid-1990s new human strains developed outside of hospitals, indicating a possible transmission route of zoonotic microorganisms colonizing farm animals to humans. We demonstrated that food and environment could be a good reservoir for bacteria, which in such environments are able to acquire and exchange genetic determinants responsible for multidrug-resistance and other virulence factors. The authors recommend not underestimating strains of different origin from that clinic, as determinants of virulence and antibiotic resistance can be evenly distributed in nature.

\section{REFERENCES}

[1] G. Makris,J. D. Wright, E. Ingham, K. T. Holland.The hyaluronatelyase of Staphylococcus aureus - a virulence factor? Microbiology, Vol.150, 2005-2013, 2004.

[2] D. Osmonov, K. S. Ozcan, I.Erdinler,S. Altay, E. Yildirim, C. Turkkan, A. Ekmekci, B. Gungor, K. Gurkan.Cardiac device-related endocarditis: 31-Years' experience, J Cardiol, Vol. 5087, 00241-00249, 2012

[3] S. Shi, X. Zhang. Interaction of Staphylococcus aureus with osteoblasts (Review),ExpTher Med, Vol. 3, 367-370,2012.

[4] R. A. de Oliveira-Calsolari, V. C. Pereira,J. P. Araújo-Júnior, M. de L. de Souza-da Cunha. Determination of toxigenic capacity by reverse transcription polymerase chain reaction in coagulase-negative staphylococci and Staphylococcus aureus isolated from newborns in Brazil,MicrobiolImmunol, Vol. 55, 394-407,2011.

[5] W. E. Kloos, T. L. Bannerman. Update on clinical significance of coagulase-negative staphylococci,ClinMicrobiol, Vol. 7, 117-140, 1994.

[6] M. G. Ammendolia,R. Di Rosa, L. Montanaro, C. R. Arciola, L. Baldassarri. Slime production and expression of slime-associatedantigen by staphylococcalclinicalisolates,J ClinMicrobiol, Vol. 37, 3235-3238, 1999.

[7] J. A. Hennekinne, M. L. De Buyser, S. Dragacci.
Staphylococcus aureus and its food poisoning toxins: characterization and outbreak investigation, FEMS Microbiol, Vol.36, 815-836, 2012.

[8] S. I.Salasia, Z. Khusnan,C.Lammler, M.Zschock.Comparative studies on pheno- and genotypic properties of Staphylococcus aureus isolated from bovine subclinical mastitis in central Java in Indonesia and Hess in Germany, J Vet Sci,Vol.5, 103-109,2004.

[9] M.U. Rasheed,A. Mohammed. Staphylococcus epidermidis: A commensal emerging as a pathogen with increasing clinical significance especially in nosocomial infections, The Internet Journal of Microbiology, Vol.3 No. 2,2007.

[10] A.Schröder, B.Schröder, B.Roppenser, S. Linder, B.Sinha, R.Fässler, M.Aepfelbacher.Staphylococcus aureusfibronecti $\mathrm{n}$ binding protein-A induces motile attachment sites and complex actin remodeling in living endothelian cells,MolBiol Cell, Vol. 17, 5198-5210, 2006.

[11] R. M. Corrigan, M. Corrigan, D. Rigby,P. Handley, T. J. Foster. The role of Staphylococcus aureus surface protein SasG adherence and biofilm formation, Microbiology, Vol. 153, 2435-2446, 2007.

[12] S.J. Peacock, C. E. Moore, A. Justice, M. Kantzanou, L. Story, K. Mackie, G. O'Neill, N.P.Day.Virulent combination of adhesion and toxin genes in natural populations of Staphylococcus aureus, Infection and Immunity,Vol. 70, 4987-4996, 2002.

[13] B.Strommenger, C. Kettlitz,G. Werner, W.Witte. Multiplex PCR assay for simultaneous detection of nine clinically relevant antibiotic resistance genes in Staphylococcus aureus, J ClinMicrobiol,Vol. 41, 4089-4094,2003.

[14] D.A.Talan, A.Krishnadasan, R. J. Gorwitz, G.E.Fosheim, B.Limbago, V. Albrecht, G.J.Moran.Comparison of Staphylococcus aureus from skin and soft-tissue infections in US Emergency department patients, 2004 and 2008, Clinical Infectious Diseases,Vol. 53, 144-149,2011.

[15] E. Ando, K. Monden, R. Mitsuhata, R. Kariyama, H. Kumon.Biofilm formation among methicillin-resistantStaphylococcusaureus isolates from patients with urinary tract infection, Acta Med Okayama, Vol. 58, 207-214, 2004.

[16] M. E. Rupp, J. S.Ulphani, P.D. Fey,D.Mack.Characterization of $S$. epidermidis polysaccharide intracellular adhesion/hemagglutinin in the pathogenesis of intravascular catheter associated infection in a rat model, Infect and Immun,Vol. 67, 2656-2659,1999.

[17] C. R. Arciola, D.Campoccia, S. Gamberini, M.Cervellati, E. Donati,L. Montanaro. Detection of slime production by means of an optimized Congo red agar plate test based on a colourimetric scale in Staphylococcus epidermidis clinical isolates genotyped for ica locus,Biomaterials, Vol. 23, 4233-4239, 2002.

[18] S. Arslan, F. Özkardes. Slime production and antibiotic susceptibility in staphylococci isolated from clinical samples,MemInstOswaldo Cruz, Vol.102, 29-33, 2007.

[19] S. de Niederhäusern, M. Bondi, P. Messi R. Iseppi, C. Sabia, G. Manicardi, I. Anacarso. Vancomycin-resistancetransferability from VanAenterococci to Staphylococcusaureus, CurrMicrobiol,; Vol. 62, 1363-1367, 2011. 
[20] J. V. Hookey,J. F. Richardson, B. D. Cookson. Molecular typing of Staphylococcus aureus based on PCR restriction fragment length polymorphism and DNA sequence analysis of coagulase gene, J ClinMicrobiol, Vol. 36, 1083-1089,1998.

[21] J. Oscarsson, K. Tegmark-Wisell, S. Arvidson. Coordinated and differential control of aureolysin (aur) and serine protease (sspA) transcription in Staphylococcus aureus by sarA, rot and agr(RNAIII), Int. J Med Microbiol, Vol. 296, 365-380, 2006.

[22] Clinical and Laboratory Standard Institutes (CLSI). Performance Standards for Antimicrobial Susceptibility Testing: Approved Standard M100-S20. Wayne, PA, USA, 2012.

[23] European Committee on Antimicrobial Susceptibility Testing (EUCAST) (2012) Clinical-breakpoints. http://www.eucast.org/clinical_breakpoints/ (Version 2.0, valid from 2012-01).

[24] M. A. Ishak, D. H. Gröschel, G. L.Mandell, R. P.Wenzel.Association of slime with pathogenicity of coagulase-negative staphylococci causing nosocomial septicemia, J ClinMicrobiol, Vol. 22, 1025-1029,1985.

[25] P. Kotilainen. Association of coagulase-negative staphylococcal slime production and adherence with the development and outcome of adult septicemias, J
ClinMicrobiol, Vol. 28, 2779-2785,1990.

[26] A. Foka, V. Chini, E. Petinaki,F. Kolonitsiou, E. D. Anastassiou, G. Dimitracopoulos, I. Spiliopoulou. Clonality of slime-producing methicillin-resistant coagulase-negative staphylococci disseminated in the neonatal intensive care unit of a university hospital,ClinMicrobiol Infect, Vol.12, 1230-1233,2006.

[27] K. K. Choudhuri, A. N. Chakrabarty.Hyaluronatelyase activity of staphylococci,Indian Journal of Experimental Biology, Vol. 7, 183-185, 1969.

[28] L. Essers, K. Radebold.Rapid and reliable identification of Staphylococcus aureus by a latex agglutination test,J ClinMicrobiol, Vol.12,641-643,1980.

[29] A. Kechrid, M. Pérez-Vázquez, H. Smaoui, D. Hariga, M. Rodríguez-Baños, A. Vindel, F. Baquero, R. Cantón, R. Del Campo. Molecular analysis of community-acquired methicillin-susceptible and resistant Staphylococcus aureus isolates recovered from bacteraemic and osteomyelitis infections in children from Tunisia, Clinical Microbiology and Infection, Vol.17, 1020-1026, 2011.

[30] D. Paludi, A. Vergara, A. R. Festino, P.Di Ciccio, C. Costanzo, M. Conter, E. Zanardi, S. Ghidini, A. Ianieri. Antimicrobial resistance pattern of methicillin-resistant Staphylococcus aureus in the food industry, $\mathrm{J}$ BiolRegulHomeost Agents,Vol.25,671-677, 2011. 